\title{
Ring-Waves Generated by Water Drops Impacting on Water Surfaces at Rest
}

\author{
Christophe Craeye, Piotr W. Sobieski, Member, IEEE, Larry F. Bliven, and Albert Guissard, Senior Member, IEEE
}

\begin{abstract}
Radar observations of the ocean surface can be affected by impacting raindrops. Ring-wave measurements are presented for drops of 2.2 and $2.8 \mathrm{~mm}$ in diameter impacting on fresh and salt water surfaces initially at rest. They are based on the observation of the mirror image of a sharp edge on the perturbed surface. The retrieved wave profiles show a rather stable characteristic wavenumber $\left(0.2 \mathrm{~mm}^{-1}\right)$ and very small wave amplitudes: the fraction of the incident kinetic energy converted into ring-waves is of the order of $1 \%$.
\end{abstract}

Index Terms - Circular surface waves, rain, wave energy.

\section{INTRODUCTION}

$\mathbf{T}$ HE IMPACT of drops on solid and liquid surfaces has been largely studied in the past. A broad review of the related phenomena is presented by Rein [1]. However, in the case of liquid target surfaces, the ring-waves generated by the impacts have been subject to rather sparse studies [2]-[6]. All those studies, except for that of Le Méhauté [3], are based on photographic and video observations, from which it is almost impossible to obtain an estimate of the height profile of the waves.

This topic becomes of special interest because of the significant effect that ring-waves produced by raindrop impacts may have on radar observations of the ocean surface [4], [7]-[10]. Indeed, at some frequencies, they can come into resonance with the incident electromagnetic waves (at centimetric wavelengths, i.e., for frequencies of the order of $10 \mathrm{GHz}$ ). In this context, it is important to study the characteristic frequencies and energy contents of the surface waves.

In view of their possible effect on the ring-waves, we recall the earliest phenomena that appear following a drop impact: when a raindrop hits a water surface, it generates a crater with a crown, which collapses to form a vertical stalk of water, which eventually subsides to spawn rings of gravity-capillary waves that propagate outward [11], [12]. Photographs of those successive events are presented in [5] and a study of the size and duration of the corresponding features is reported in [6]. A large fraction of the drop energy is certainly transformed into vortex rings propagating vertically through the water [13], while the energy fraction transferred horizontally by the ringwaves is probably smaller, but has never been quantified [14].

Manuscript received November 15, 1997; revised April 9, 1999.

C. Craeye, P. W. Sobieski, and A. Guissard are with the Universite Catholique de Louvain, B-1348 Louvain-la-Neuve, Belgium.

L. F. Bliven is with NASA/GSFC/WFF, Laboratory for Hydrospheric Processes, Wallops Island, VA 23337 USA.

Publisher Item Identifier S 0364-9059(99)06263-9.

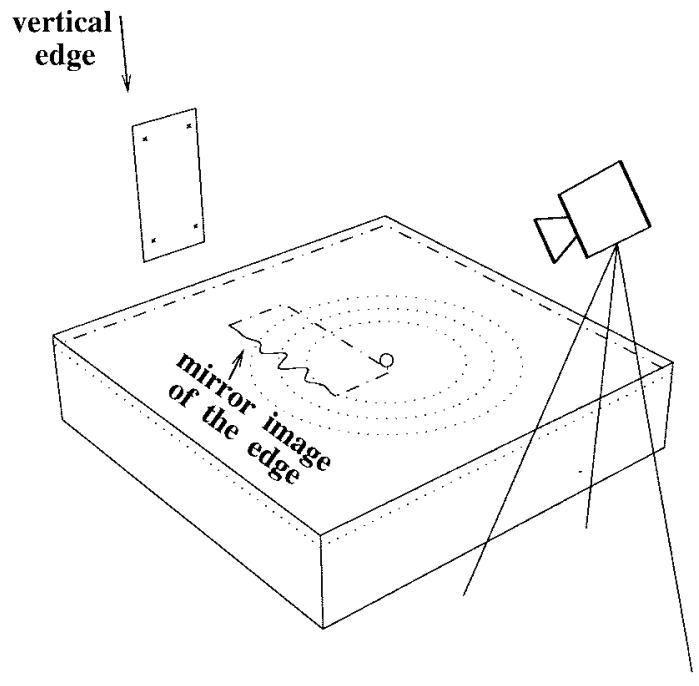

Fig. 1. Experimental setup.

In this paper, we present a measurement technique for ringwaves based on slope estimation through the observation of the image, on the perturbed surface, of the edge of a sharp object. Special care is given to the effect of noise on the integration of slope profiles, and we provide estimates of total energy and characteristic frequency of the ring-waves.

The experimental setup is presented in Section II and the data are analyzed in Section III, with the help of basic equations governing the propagation of circular gravity-capillary waves. A discussion follows in Section IV, where the results are compared with the theoretical model developed by Le Méhauté [3]. Finally, we compare the ring-wave energy with the kinetic energy of the impinging drop.

\section{Measurement Technique}

Surface waves are often measured with capacitive wire probes that present the advantage of measuring directly the surface height. However, they give only one-point observations and present generally a sensitivity of a few tenths of millimeters, which is insufficient to observe the phenomenon with which we are dealing. To overcome those difficulties, we adopted an optical technique: the ring-waves have been observed indirectly through their effect on the mirror image of a vertical line on the water surface. The experimental setup is presented in Fig. 1: the vertical line corresponds to the left edge of a white page placed on a dark background, and 
the scene is observed with a video camera. The center circle represents the impact point of the drop.

The positions of the page and of the camera were measured accurately, and four reference points of known position were drawn on the page (crosses on the figure). We considered the image as a central projection of the scene, whose parameters were estimated through the knowledge of the position of the reference points. From there, a correspondence could be established between each pixel of the mirror image and the horizontal position of every point observed on the water surface. This was done neglecting, at this stage, the vertical position of the points, because of the very small vertical displacements of the water surface (verified later on in Section III-D).

This allows the determination of the horizontal coordinates of the impact point and of the distorted image of the edge. For each point of the edge image, the local normal can be estimated using two pieces of information.

- As seen from the camera, the observed point corresponds to the image of a point of the vertical edge.

- Due to the cylindrical symmetry of the wave, the local normal is in the vertical plane containing the impact point.

From this, a slope profile is calculated along the ring radius. Details of this technique are given in Appendix I.

The horizontal dimensions of the tank were $1.1 \times 1.1 \mathrm{~m}$ and the water depth was $20 \mathrm{~cm}$. The experiments were carried out with tap water (measured surface tension: 70 dynes $\cdot \mathrm{cm}^{-1}$ ) and with salt water, whose salinity was 34 parts per thousand (measured surface tension: 60 dynes $\cdot \mathrm{cm}^{-1}$ ), which corresponds to the mean salinity of sea water. The water surface was cleaned every $30 \mathrm{~min}$ on average.

The drops were made of tap water and were produced by two different hypodermic needles, corresponding to drop diameters of 2.2 and $2.8 \mathrm{~mm}$ (assuming an equivolumetric spherical shape). They fell from three different heights (1, 3, and $5.5 \mathrm{~m}$ ), which correspond to approximately $55 \%, 81 \%$, and $93 \%$ of terminal velocity [15].

The camera used was an analog SONY camera, with a frame rate of 25 images per second. With this kind of video device, the blurring of the images may have two origins: the fast motion of the wave and a defocusing effect related to the slight curvature of the observed surface. Those effects were made negligible by selecting a high shutter speed (1/150 s) and a small diaphragm aperture, along with a strong illumination $(1 \mathrm{~kW})$.

\section{DATA PROCESSING}

\section{A. Slope Profiles}

For each particular experiment, the edge image was observed at three successive moments, separated by $3 / 25$ s (every 3 frames). Each image was digitized in a $784 \times 576$ pixels frame, where the edge of the page was detected. From there, using the methodology mentioned above, a slope profile was determined. Fig. 2 gives an example of such a slope profile for a 2.8-mm drop falling from $5.5 \mathrm{~m}$ into fresh water.

The accuracy of the measurements is essentially limited by the pixel resolution of the camera and by the accuracy

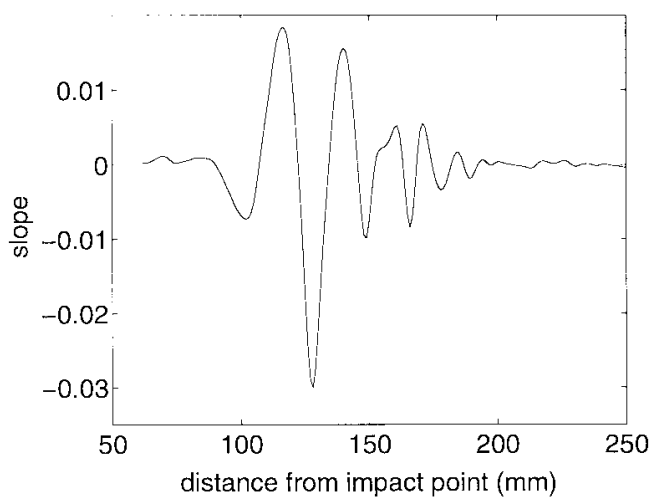

Fig. 2. Slope profile of a ring-wave packet produced $840 \mathrm{~ms}$ after impact by a $2.8-\mathrm{mm}$ drop falling into fresh water from a $5.5-\mathrm{m}$ height.

of the determination of horizontal coordinates. The effect of limited resolution was observed in the case of a flat surface, for which the detected slope presented a zero mean and a standard deviation of $0.5 \times 10^{-3} \mathrm{~mm} / \mathrm{mm}$. This corresponds, for the slope profiles, to a signal-to-noise ratio (SNR) of the order of $20 \mathrm{~dB}$ for the 2.2-mm drops and $26 \mathrm{~dB}$ for the 2.8-mm drops. The errors in horizontal coordinates stem essentially from the nonlinear aberrations of the camera and were smaller than $2 \%$. The consequences of these uncertainties will be discussed below.

\section{B. Wave Propagation}

Basic relationships for the propagation of circular gravitycapillary surface waves are presented in Appendix III. They are needed to check the dispersion and dissipation of the waves and they also provide a simple method to estimate the height profiles and wave energy.

Let $\eta\left(r, t_{1}\right)$ be the height profile of the circular wave versus the ring radius $r$ at a time $t_{1}$ for which the perturbed ring is already far from the source. We may denote $A_{0}(k)$ as the complex Hankel transform of $\eta\left(r, t_{1}\right)$, which we defined (see Appendix II) as

$$
\begin{aligned}
A_{0}(k) & =\mathbf{H}\left\{\eta\left(r, t_{1}\right)\right\} \\
& =1 / 2 \int_{0}^{\infty} \eta\left(r, t_{1}\right)\left[J_{0}(k r)-j Y_{0}(k r)\right] r d r
\end{aligned}
$$

where $J_{0}$ and $Y_{0}$ are the first kind Bessel and Neumann functions of zeroth order.

If we neglect the dissipation, it is possible to show (see Appendix III) that the wave shape at another instant $t$ can be modeled in very good approximation by

$$
\begin{aligned}
\eta(r, t)= & \Re\left\{\int_{0}^{\infty} A_{0}(k) e^{-j \omega\left(t-t_{1}\right)}\right. \\
& \left.\cdot\left[J_{0}(k r)+j Y_{0}(k r)\right] k d k\right\},
\end{aligned}
$$

i.e., the wave shape is represented by a superposition of progressive wave components characterized by a wavenumber $k$.

The link between the time pulsation $\omega$ and the wavenumber $k$ is given by the dispersion relationship for gravity-capillary 
waves

$$
\omega^{2}=g k+\tau / \rho k^{3}
$$

where $g$ is the acceleration of gravity, $\tau$ is the surface tension, and $\rho$ is the density of water.

Approximation (2) is very good provided that the $k r$ product is large, i.e., the ring-wave radius must be large compared to its characteristic wavelengths. This condition is fulfilled in the case of our observations. Indeed, as explained below, typical values of the distance from the origin and of the wavelength are 110 and $30 \mathrm{~mm}$, respectively, which gives a $\mathrm{kr}$ product of 23 .

\section{Dissipation and Dispersion}

In view of the rather low dissipation rate, the wave damping coefficients may be simply superimposed to the solution described above, i.e., we apply a perturbation model, also used by Le Méhauté [3]. Following Van Dorn [16], we consider two sources of dissipation: 1) the viscous dissipation and 2) the wave suppression by the surface film that appears because of the practically unavoidable surface contaminants. Both of those dissipation sources are characterized by an exponential damping coefficient, $D(k)=e^{-\gamma t}$, where $\gamma=2 \nu k^{2}$ for the viscous dissipation and $\gamma=k \sqrt{\omega(k) \nu / 8}$ for the effect of the surface film in case of full contamination, where $\nu$ is the molecular viscosity of water $\left(1 \mathrm{~mm}^{2} \cdot \mathrm{s}^{-1}\right.$ both for fresh and salt water).

In the-rather narrow-wavenumber range that we will consider here, we may approximate both $\gamma$ exponents by a quadratic law versus wavenumber. Therefore, we modeled the dissipation by one single exponent: $D(k)=e^{-2 \nu_{\text {cqu }} k^{2} t}$, where $\nu_{\text {equ }}$ is an equivalent viscosity. If we consider a central wavenumber of $k=0.2 \mathrm{~mm}^{-1}$ (see below), we find $\nu_{\text {equ }}=$ $7.28 \mathrm{~mm}^{2} \cdot \mathrm{s}^{-1}$.

From (3), we may also predict a strong dispersion and, hence, a rapid change of the shape of the elevation wave. This is effectively observed.

In order to check the quality of the measurements, we compared the surface slope profile at a given moment $t$ with that predicted using the one observed at a previous time $t_{1}$ (3/25 s earlier).

Developments of Sections II-B and II-C showed that, from time $t_{1}$ to time $t$, the complex Hankel transform $A_{0}(k)$ of the wave profile is multiplied by the phase factor related to the propagation, and by the exponential factor standing for the dissipation.

If we approximate $\mathbf{H}\{d z(r) / d r\}$ by $j k \mathbf{H}\{z(r)\}$ (see Appendix II), the same transformations may be applied to the complex Hankel transform $B_{0}(k)$ of the slope profile.

Hence, an estimate of $d \eta / d t$ at time $t$ is given by

$$
\begin{aligned}
d \eta(r, t) / d r=\Re & \left\{\int_{0}^{\infty} B_{0}(k) e^{-j \omega\left(t-t_{1}\right)} e^{-2 \nu_{e q u} k^{2}\left(t-t_{1}\right)}\right. \\
\cdot & {\left.\left[J_{0}(k r)+j Y_{0}(k r)\right] k d k\right\} }
\end{aligned}
$$

and it can be compared with the experimental profile at that moment. (a)

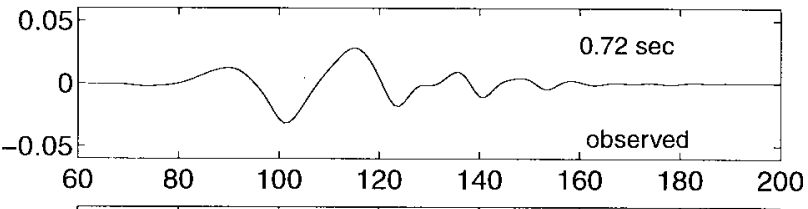

(b)

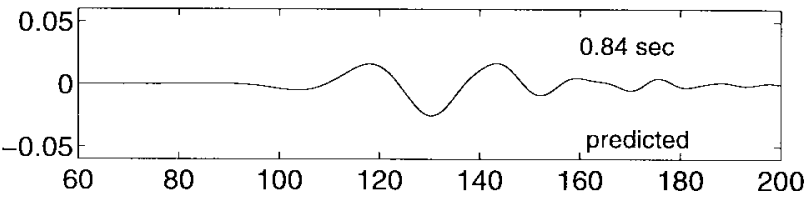

(c)

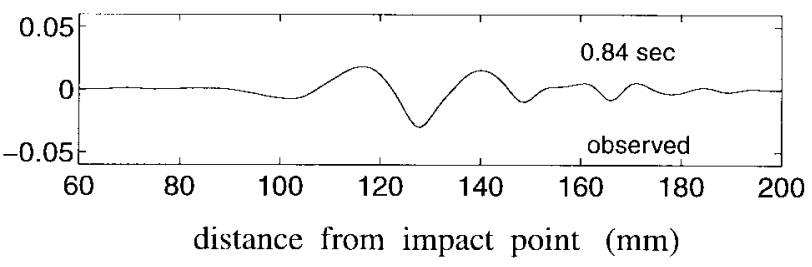

Fig. 3. Slope profiles for a $2.8-\mathrm{mm}$ drop falling from a 5.5-mm height into fresh water: (a) observed at $720 \mathrm{~ms}$, (b) predicted for $840 \mathrm{~ms}$ using data obtained at $720 \mathrm{~ms}$, and (c) observed at $840 \mathrm{~ms}$.

(a)

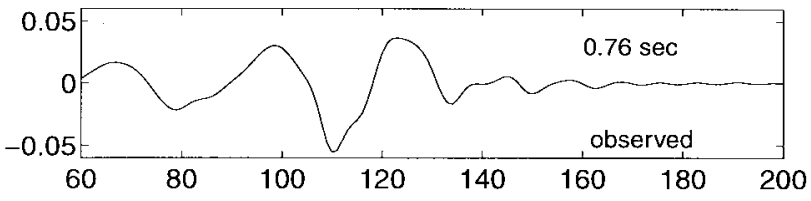

(b)

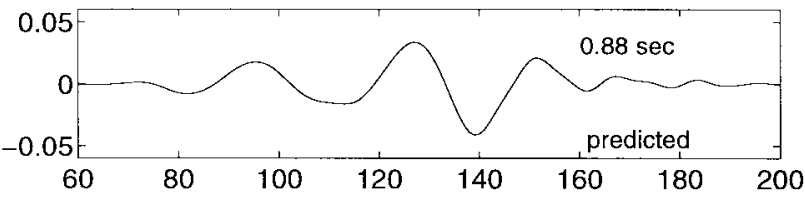

(c)

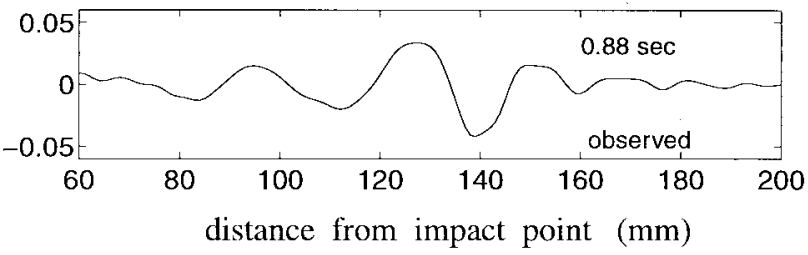

Fig. 4. Slope profiles for a 2.8-mm drop falling from a 5.5-mm height into salt water: (a) observed at $760 \mathrm{~ms}$, (b) predicted for $880 \mathrm{~ms}$ using data obtained at $760 \mathrm{~ms}$, and (c) observed at $880 \mathrm{~ms}$.

Two examples of such comparisons are shown in Figs. 3 and 4, where we observe a good agreement between (b) predicted and (c) measured profiles, both for fresh and salt water. This shows that the shape evolution is satisfactorily modeled by using the dispersion relationship (3) and the measured values of the surface tension. For example, according to a leastsquares error criterium, the differences between the shapes in Fig. 3(b) and (c) in terms of amplitude and horizontal position are only $2 \%$ and $3 \mathrm{~mm}$, respectively. The corresponding leastsquares error is less than half that obtained when the shapes in Fig. 3(a) and (c) are compared. The agreement is even better for the salt water case shown in Fig. 4. This supports the good quality of the measuring system.

\section{Wave Characteristics}

The adopted measurement technique does not allow us to observe the ring-waves at very early instants of development. Since the best observation instant is around $800 \mathrm{~ms}$ after 


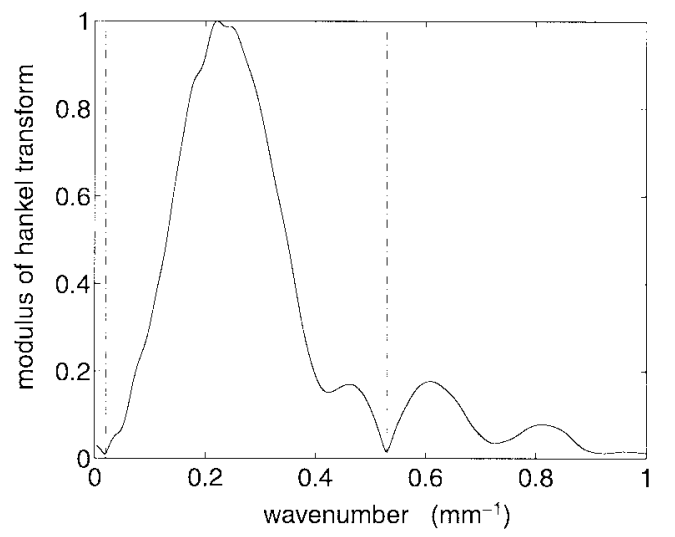

Fig. 5. Modulus of the (normalized) Hankel transform of the slope profile measured for a $2.8-\mathrm{mm}$ drop falling from a $5.5-\mathrm{m}$ height into fresh water. The vertical lines represent the filtering limits.

impact, we characterized each observed ring-wave at that particular time. Estimates of the wave profile at other instants can easily be obtained using the propagation law described above [see (2)]. However, two important comments need to be made.

First, the video frames were not synchronized with the instant of impact, and the frame rate was rather low $(25$ images/s). This leads to an uncertainty of $40 \mathrm{~ms}$ on the absolute timing of the selected image. This is an important source of difference between repeated measurements of the same kind.

Second, the integration of the slope profiles must be performed with caution because of the possible propagation of errors that can lead to a drift of the mean level of the height profiles. The adopted solution consisted of a filtering based on the elimination of high-frequency components related to quantization effects (due to the finite pixel size of the images) and of low-frequency components that can be strongly enhanced through the integration process. Therefore, the complex Hankel transform of the slope profile was limited to a band located between deep local minima of the slope spectrum (modulus of $\mathbf{H}\{d \eta(r) / d r\}$ ) in the vicinity of $0.05 \mathrm{~mm}^{-1}$ and $0.5 \mathrm{~mm}^{-1}$, as shown in Fig. 5. From the knowledge of the noise level, obtained for a surface at rest, we estimated that the maximum drift of the mean level after filtering yields an error of about $0.02 \mathrm{~mm}$.

The height profile was obtained by considering that $\mathbf{H}\{d \eta(r) / d r\} \simeq j k \mathbf{H}\{\eta(r)\}$, where $\mathbf{H}$ denotes the complex Hankel transform. This is verified as a good approximation if the $k r$ product is large (see Appendix II), which is the case for the distances from source and the wavenumbers considered here.

So, in the wavenumber band selected by the filtering, an estimate of $\mathbf{H}\{\eta(r)\}$ was obtained by dividing the complex Hankel transform of the slope profile by $j k$ and by keeping it to zero elsewhere. The height profile was then obtained through (see Appendix II)

$$
\eta(r) \simeq \Re\left\{\int_{0}^{\infty} \mathbf{H}\{\eta(r)\}\left[J_{0}(k r)+j Y_{0}(k r)\right] k d k \cdot\right\}
$$

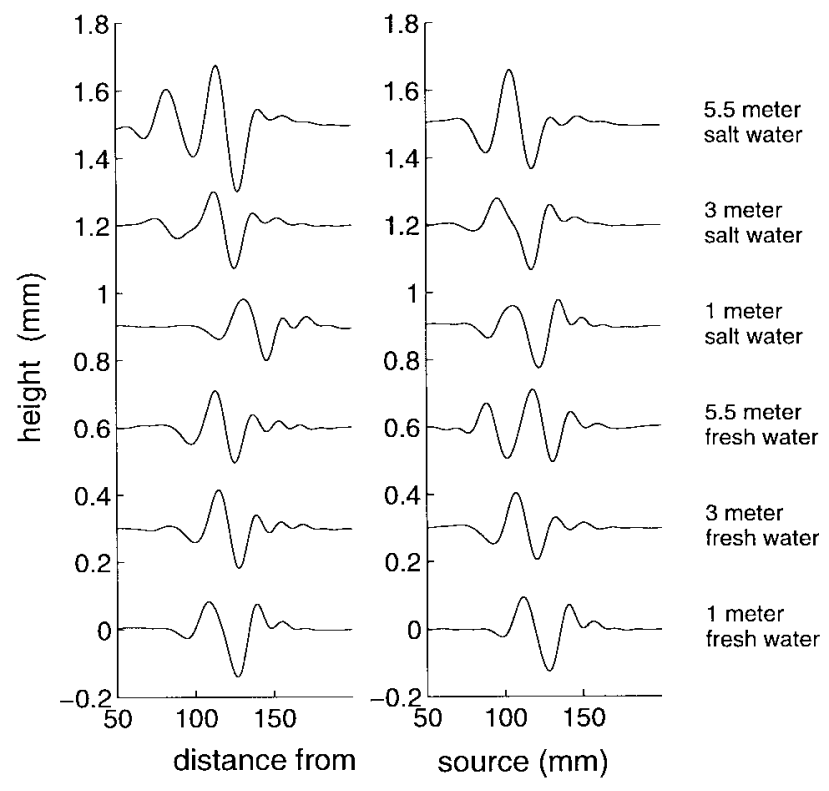

Fig. 6. Height profiles obtained for 2.8-mm drops falling from different heights into fresh and salt water. For each type of conditions, the results of two observations are represented (one in each column). The profiles are shifted vertically by $0.3 \mathrm{~mm}$ one to each other.

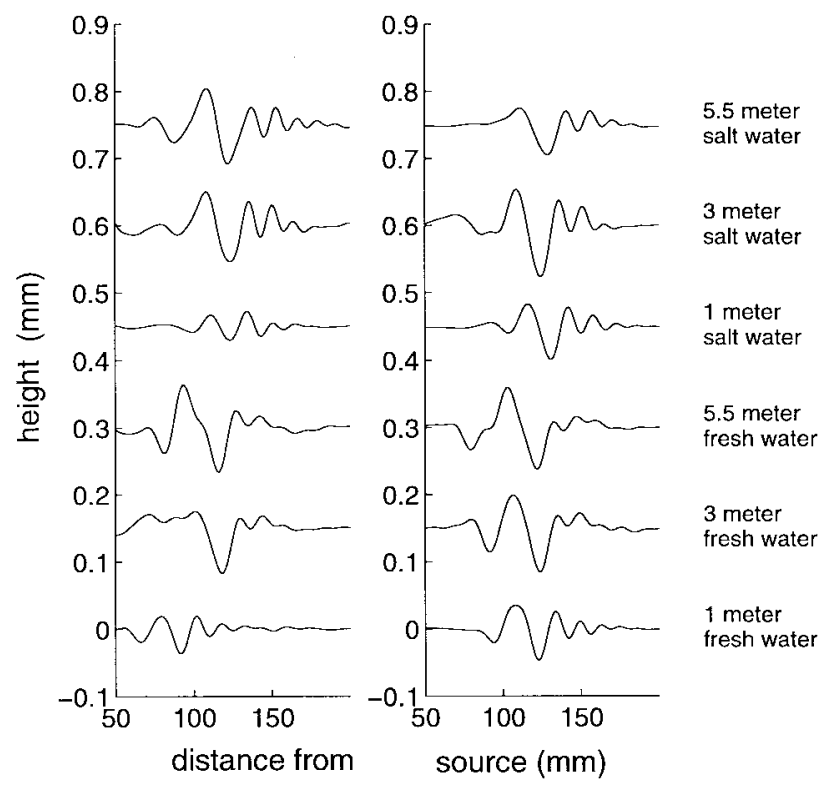

Fig. 7. Height profiles obtained for 2.2-mm drops falling from different heights into fresh and salt water. For each type of conditions, the results of two observations are represented (one in each column). The profiles are shifted vertically by $0.15 \mathrm{~mm}$ one to each other.

Note also that $\mathbf{H}\{\eta(r)\}=0$ for $k=0$, which guarantees volume conservation around the zero-level

$$
\int_{S} \eta d S=2 \pi \int_{0}^{\infty} \eta(r) r d r=4 \pi \Re\left\{\mathbf{H}\{\eta(r)\}_{k=0}\right\}=0 .
$$

Several examples are presented in Figs. 6 and 7 for 2.2- and 2.8-mm drops, where two height profiles are given for each set of experimental conditions (depending on fall height and water salinity). We observe the very small amplitudes of the waves, especially for the $2.2-\mathrm{mm}$ drops. The drift related to 


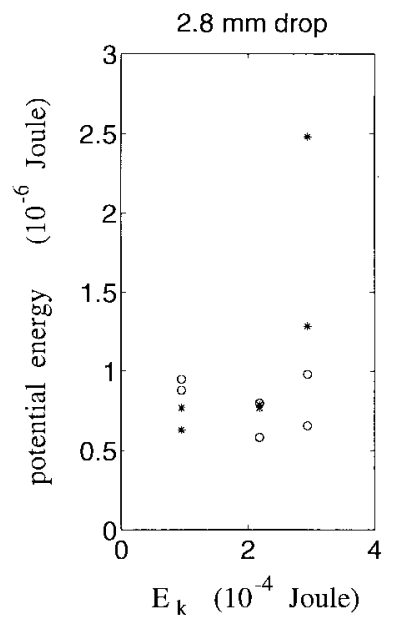

(a)

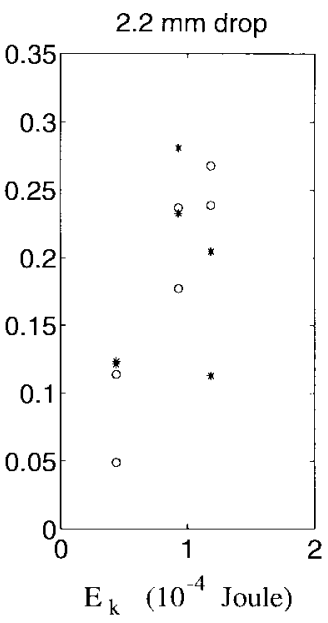

(b)
Fig. 8. Potential energy of the ring-waves near $800 \mathrm{~ms}$ after impact, as a function of the kinetic energy of the impacting drops. Circles indicate fresh water and stars indicate salt water for (a) $2.8-\mathrm{mm}$ and (b) $2.2-\mathrm{mm}$ drops.

the integration of noise is visible on the plots but is limited to an acceptable value.

The total potential energy of the wave is equal to

$$
E_{p}=\frac{\rho g}{2} \int_{0}^{2 \pi} \int_{0}^{\infty} \eta(r)^{2} r d r d \phi \simeq 2 \rho g \pi \int_{0}^{\infty} S(k) d k
$$

with $S(k)=|\mathbf{H}\{\eta(r)\}|^{2} k$ (second property of the complex Hankel transform, see Appendix II).

We also define the characteristic wavenumber $k_{m}$ as the following spectral mean:

$$
k_{m}=\frac{\int_{0}^{\infty} S(k) k d k}{\int_{0}^{\infty} S(k) d k} .
$$

Figs. 8 and 9 show $E_{p}$ and $k_{m}$ for the 2.8- and 2.2-mm drops; stars indicate the salt water experiments and circles indicate the fresh water cases. These quantities are displayed as functions of the kinetic energy of the impinging drops, which is proportional to $d^{3} v\left(h_{i}\right)^{2}$, where $d$ is the drop diameter and $v$ is the impacting velocity for a drop of diameter $d$ and falling height $h_{i}(1,3$, or $5.5 \mathrm{~m})$.

In Fig. 9, we observe a rather stable value of the characteristic wavenumber, around $0.2 \mathrm{~mm}^{-1}$, while the spread of the energy values (Fig. 8) is larger. The effect of the fall height on this latter parameter is not very clear, while a strong difference is visible between the two drop sizes. For similar conditions, there is at least a factor 4 between the potential energy of ring-waves related to $2.8-\mathrm{mm}$ drops and that related to $2.2-\mathrm{mm}$ drops, while there is almost a factor 2 between corresponding incident kinetic energies.

\section{DISCUSSION}

For each type of experiment, the observation was repeated twice: the height profiles, presented in Figs. 6 and 7, show more or less similar results for repeated experiments. Note, however, that the exact shapes of successive events cannot be

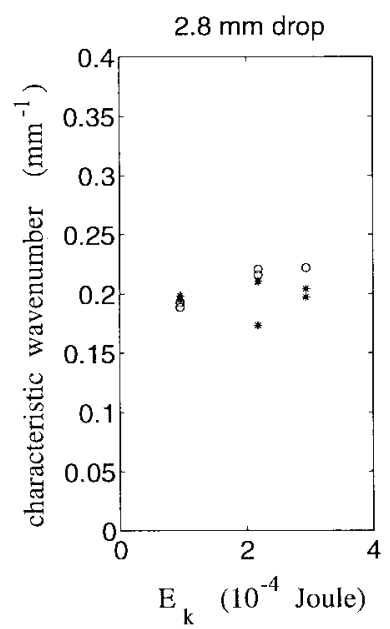

(a)

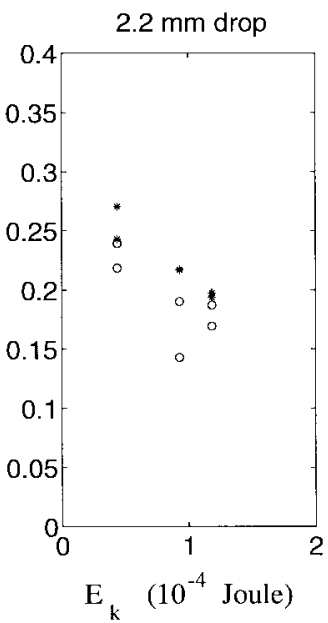

(b)
Fig. 9. Characteristic wavenumbers near $800 \mathrm{~ms}$ after impact as a function of the kinetic energy of the impacting drops. Circles indicate fresh water and stars indicate salt water for (a) 2.8- $\mathrm{mm}$ and (b) 2.2-mm drops.

compared. A first reason for this is the combination of the limited timing accuracy $(40 \mathrm{~ms})$ and the strong dispersion of the waves. A second reason is related to the small fraction of the drop energy contained in the ring-waves (see below), which explains the natural variability of the ring-wave shapes.

The experimental results can be summarized as follows.

- There is no significant difference between fresh water and salt water experiments.

- The characteristic wavenumber $\left(k_{m}\right)$ always lies near 0.2 $\mathrm{mm}^{-1}$, which corresponds to a wavelength of almost 3 $\mathrm{cm}$, i.e., slightly larger than what can be seen on the height profiles. The difference is due to the particular definition of $k_{m}$ and to the rather asymmetric shape of the height spectra.

- For a given time, the wavelength of the ring-waves decreases with the ring radius. This can sometimes be observed on the height profiles and was particularly visible on the video images at earlier instants: a highfrequency wave precedes the main rings, but its fine structure could not be measured because of its very rapidly decreasing amplitude.

- The amplitudes are very small: $800 \mathrm{~ms}$ after impact, the maximum height appears around $13 \mathrm{~cm}$ from the point of impact and is of the order of $0.1 \mathrm{~mm}$ for the $2.8-\mathrm{mm}$ drop and $0.05 \mathrm{~mm}$ for the 2.2-mm drop. The fall height-and thus the impacting velocity-did not show a strong effect on the ring-wave amplitude.

- The width of the perturbed ring is near $6 \mathrm{~cm}$.

From the present study, it is impossible to give, as it was proposed in [4], a model for the shape of the wave packet because of the strong dispersion. However, the evolution of the potential energy of the wave can be approximated by considering the $e^{-2 \nu_{e q u} k^{2} t}$ factor described in Section II, where $k$ is taken near the characteristic wavenumber: $k_{m} \simeq$ $0.2 \mathrm{~mm}^{-1}$.

It is interesting to compare our experimental results with the theoretical study presented by Le Méhauté [3]. This 
author models the drop impact as an additional pressure on the surface, represented by a Dirac function in time and extending over an area limited by the drop size. From there, he finds that the multiplicity of rings observed at a distance are solely the results of linear dispersion from the initial nonoscillatory movement. He also finds that these waves have a narrow range of wavenumbers around $k_{b}$, the wavenumber that corresponds to the minimum group velocity of the surface waves, i.e., the wavenumber range is only dictated by the fluid characteristics. $k_{b}$ is equal to $0.143 \mathrm{~mm}^{-1}$ for a surface tension $\tau=74$ dynes $\cdot \mathrm{cm}^{-1}$ and is proportional to $\tau^{-1 / 2}$.

Our experiments show a sensibly higher $\left(0.2 \mathrm{~mm}^{-1}\right)$ characteristic wavenumber and complex phenomena at the point of impact: generation of a crown, a crater, and a single or multiple stalk [5]. Video observations show that the collapse of those successive events is also an important source of ring-waves [6]. This means that the timing of the outgoing ring-waves can be strongly influenced by the timing of the movements at the point of impact. For example, the time separating the collapse of the crown and that of the stalk is of the order of $100 \mathrm{~ms}$, which, combined with a phase velocity of $250 \mathrm{~mm} \cdot \mathrm{s}^{-1}$ gives rise to a wavelength of $25 \mathrm{~mm}$, while the main wavelength predicted by Le Méhauté is equal to $\lambda_{b}=2 \pi / k_{b}=43 \mathrm{~mm}$.

Finally, we can compare the total energy of the ring-waves to the kinetic energy $E_{k}$ of the impacting drop. Let us make this comparison for the case of the $2.8-\mathrm{mm}$ drop and for a fall height of $5.5 \mathrm{~m}$. In this case, $E_{k}=3 \cdot 10^{-4} \mathrm{~J}$ and the ring-wave energy can be grossly estimated assuming that:

- the kinetic energy of the wave at a given moment is equal to the sum of the potential energy and that related to the surface tension;

- the ratio between the energy related to the surface tension and the potential energy is equal to $(\tau / \rho g) k_{m}^{2}$, where $k_{m}$ is the characteristic wavenumber (these two first assumptions are exact for linear monochromatic waves [2]);

- the fraction of energy dissipated since the instant of impact is given by $1-e^{-2 \nu_{e q u} k^{2} T}$, where $T$ is the time of observation ( $T=0.8 \mathrm{~s}$ here).

Using those assumptions and the results given in Fig. 8 for $T=0.8 \mathrm{~s}$, the total generated ring-wave energy is estimated to be $E_{t, r}=5.9 \cdot 10^{-6} \mathrm{~J}$, which is only $2 \%$ of the incident kinetic energy $E_{k}$.

For 2.2-mm drops falling from a 5.5-m height, the fraction of kinetic energy transferred into ring-waves is even smaller: it is less than $1 \%$. In other terms, the energy transfer ratio seems to depend strongly on the drop size. This could be related to the impulsive interaction between the drop and the target surface. Indeed, when the two drop sizes are compared, the ratio of the ring-wave amplitudes (average value: 2.56 ) is close to the ratio of the drop momentums (average value: 2.26), which is coherent with the amplitude behavior of the solution obtained theoretically by Le Méhauté, assuming a very idealized interaction.

The very small fractions of energy involved in the ringwaves indicate that nearly all of the incident energy is dissi- pated in the bulk of the liquid, near the point of impact, most probably after transformation in vorticity, which can be either coherent or turbulent, depending on the entry Reynolds number of the drop [13]. The generation mechanisms of vortices and their different regimes are not yet quite well identified and they are out of the scope of this paper. For further information on this topic, we invite the interested reader to refer to a review paper by Rein [1]. Finally, we should also mention that the damping of sea waves by heavy rain is often attributed to the generation of vortex rings by the impacting drops [17], [18]. Besides the effect of ring-waves, the damping of the sea surface by heavy rain may also explain several anomalous radar returns obtained from space [9], [19].

\section{CONCLUSION}

A measurement technique based on slope determination has enabled us to obtain good estimates of the characteristic amplitudes, frequencies, and durations of ring-waves produced by drop impacts on water surfaces. Three different fall heights have been considered, the highest one corresponding to a near-terminal fall velocity. Both fresh and salt target surfaces were used; however, no systematic effect of the salinity could be delineated. The experiments showed ring-waves whose characteristic wavenumber was around $0.2 \mathrm{~mm}^{-1}$ and very small amplitudes near $0.8 \mathrm{~s}$ after impact (of the order of $0.1 \mathrm{~mm})$. What makes these waves visible to the human eye is probably the strong modulation that very small slopes induce on light reflection. This effect was exploited here to characterize the ring-waves.

A major conclusion of this study is that only a very small fraction of the incident kinetic energy is converted into ring-waves, the rest being most probably transformed into vorticity, propagating vertically through the liquid. Finally, we also observed that the ring-wave energy presents a very strong dependence on the drop size; in other words, it cannot be considered as simply proportional to the incident kinetic energy.

\section{APPENDIX I \\ SLOPE EstimATION TECHNIQUE}

The slope profile of a ring-wave is determined by observing the mirror image on the water surface of a vertical edge.

Let us define an orthonormal coordinate system where the edge corresponds to the vertical axis.

1) $\eta(x, y)$ is the surface height at a point of horizontal coordinates $x$ and $y$.

2) $\bar{x}=(x, y, 0)$ is a point of the image of the edge, for which the height has been neglected.

3) $\bar{x}_{1}=\left(x_{1}, y_{1}, 0\right)$ is the impact point.

4) $\bar{w}=(0,0, h)$ is a point on the vertical edge, i.e., the edge corresponds to the vertical axis of the coordinate system.

5) $\bar{o}=\left(x_{o}, y_{o}, z_{o}\right)$ is the focal center of the camera lens.

At a point $\bar{x}$ of the reflected edge, the local slopes can be estimated as follows.

- As seen from the camera, $\bar{x}$ is the image of a source point $\bar{w}$ on the edge. Snell's law stipulates that the angles $\alpha$ and 


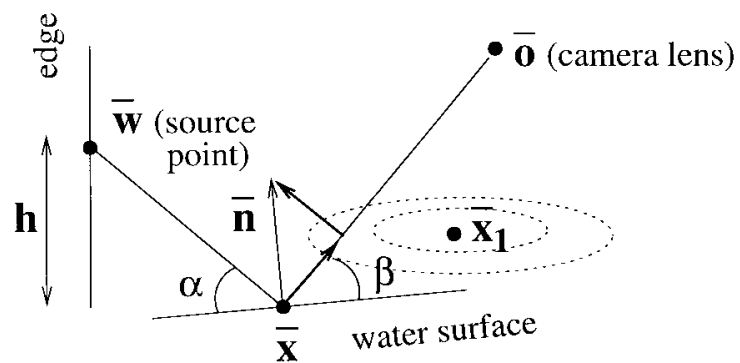

Fig. 10. Geometrical configuration in the reflection plane.

$\beta$ (Fig. 10) are equal, from which the following property holds:

$$
\frac{\bar{o}-\bar{x}}{\|\bar{o}-\bar{x}\|}+\frac{\bar{w}-\bar{x}}{\|\bar{w}-\bar{x}\|}=\bar{n}
$$

where $\bar{n}$ is normal to the surface (but does not have a unit length). In the following, we will also denote it by its rectangular coordinates $(a, b, c)$. This vector cannot be immediately estimated because the position $\bar{w}$ (i.e., the height $h$ ) of the source point is unknown.

This problem can be solved using another fact:

- Given the circular symmetry of the waves, the surface gradient is oriented toward the impact point, i.e., it is parallel to the vector $\left(x_{1}-x, y_{1}-y\right)$. Hence

$$
\frac{\partial \eta / \partial x}{\partial \eta / \partial y} \equiv \frac{-a / c}{-b / c}=\frac{x_{1}-x}{y_{1}-y}
$$

Using (9) and (10), some algebra yields the height $h$ of the source point as

$$
h=\sqrt{D^{2}-x^{2}-y^{2}}
$$

with

$$
D=\frac{\left(y x_{1}-x y_{1}\right) \sqrt{\left(x_{o}-x\right)^{2}+\left(y_{o}-y\right)^{2}+z_{o}^{2}}}{\left(x_{1}-x\right)\left(y_{o}-y\right)-\left(x_{o}-x\right)\left(y_{1}-y\right)} .
$$

Then, the local normal is obtained using (9), hence $\partial \eta / \partial x$ and $\partial \eta / \partial y$ are found. The derivative along the ring radius $\left(r=\left\|\bar{x}-\overline{x_{1}}\right\|\right)$ is

$$
\partial \eta / \partial r=\left[\partial \eta / \partial x\left(x-x_{1}\right)+\partial \eta / \partial y\left(y-y_{1}\right)\right] / r .
$$

So, for every point of the edge image, $r$ and $\partial \eta / \partial r$ can be calculated and, hence, the slope profile of the ring-wave along its radius.

\section{APPENDIX II COMPleX HANKel TRANSFORM}

Given a real function $z(r)$, its real Hankel transform $H_{i}\{z(r)\}(k)$ associated to the Bessel function of first kind and order $i$ is defined by

$$
H_{i}\{z(r)\}(k)=\int_{0}^{\infty} z(r) J_{i}(k r) r d r .
$$

Two properties of this transform are [20]:

Inverse transform:

$$
z(r)=\int_{0}^{\infty} H_{i}\{z(r)\}(k) J_{i}(k r) k d k .
$$

Parseval theorem:

$$
\int_{0}^{\infty} f(r) g(r) r d r=\int_{0}^{\infty} H_{i}\{f(r)\} H_{i}\{g(r)\} k d k
$$

Let us now define the zeroth-order complex Hankel transform $\mathbf{H}\{z(r)\}=A(k)$ of a real function $z(r)$ as

$$
\mathbf{H}\{z(r)\}=A(k) \stackrel{\text { def }}{=} 1 / 2 \int_{0}^{\infty} z(r)\left[J_{0}(k r)-j Y_{0}(k r)\right] r d r .
$$

Henceforth, we will assume that the real functions $z(r)$ that are considered present significant values only for $r$ large compared to their characteristic wavelengths.

This means that the integrand of (16) has a significant contribution only when $\mathrm{kr} \gg \mathbf{1}$. Under this condition, the properties following hereafter are valid. They will be proved with the help of the following approximation, valid for large arguments $x$ :

$$
Y_{0}(x) \simeq J_{1}(x) \simeq \sqrt{\frac{2}{\pi x}} \sin (x-\pi / 4)
$$

Property 1:

$$
f(r)=\Re\left\{\int_{0}^{\infty} A(k)\left[J_{0}(k r)+j Y_{0}(k r)\right] k d k\right\} \simeq z(r) .
$$

Indeed, if we replace $A(k)$ by its expression, we have

$$
\begin{aligned}
f(r) \simeq & \int_{0}^{\infty}\left\{\frac{1}{2} \int_{0}^{\infty} z\left(r^{\prime}\right) J_{0}\left(k r^{\prime}\right) r^{\prime} d r^{\prime}\right\} J_{0}(k r) k d k \\
& +\int_{0}^{\infty}\left\{\frac{1}{2} \int_{0}^{\infty} z\left(r^{\prime}\right) J_{1}\left(k r^{\prime}\right) r^{\prime} d r^{\prime}\right\} J_{1}(k r) k d k \\
= & z(r)
\end{aligned}
$$

where $Y_{0}$ has been replaced by $J_{1}$ and the inversion property of the real Hankel transform has been used.

Property 2:

$$
\int_{0}^{\infty}|f(r)|^{2} r d r \simeq 2 \int_{0}^{\infty}|\mathbf{H}\{f(r)\}|^{2} k d k
$$

By using twice the Parseval theorem (15), the left term of (19) can be written as

$$
1 / 2\left\{\int_{0}^{\infty}\left(H_{0}\{f(r)\}\right)^{2} k d k+\int_{0}^{\infty}\left(H_{1}\{f(r)\}\right)^{2} k d k\right\} .
$$

On the other hand, the right term of (19) equals

$$
\begin{aligned}
2 \int_{0}^{\infty} & \left(1 / 2 \int_{0}^{\infty} f(r) J_{0}(k r) r d r\right)^{2} \\
& +\left(1 / 2 \int_{0}^{\infty} f(r) Y_{0}(k r) r d r\right)^{2} k d k
\end{aligned}
$$

which corresponds to the left term of (19) if we replace $Y_{0}$ with $J_{1}$. 
(a)

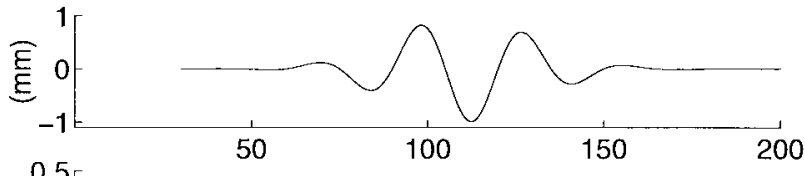

(b)

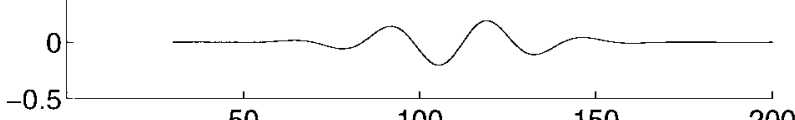

(c)

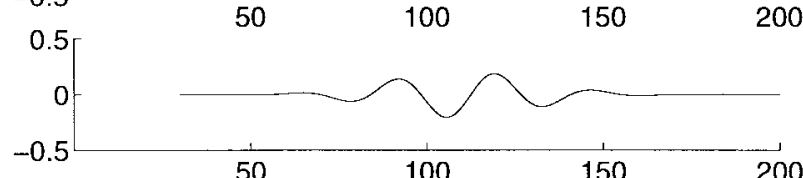

(d)

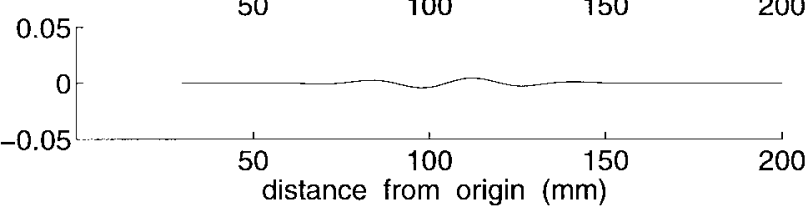

Fig. 11. (a) A function of the radius $r$, (b) its derivative, (c) an estimate of its derivative using the approximate properties of the complex Hankel transform, and (d) the difference between $d z(r) / d r$ and its estimate.

Property 3: Let us assume, in addition to the above cited condition, that $\lim _{r \rightarrow 0} z(r) Y_{0}(k r)=0$ and $\lim _{r \rightarrow \infty} z(r) r=$ 0 . This gives a sufficient set of conditions to obtain the following property:

$$
\mathbf{H}\{d z(r) / d r\} \simeq j k \mathbf{H}\{z(r)\} .
$$

Indeed, through integration by parts, we obtain

$$
\begin{aligned}
2 \mathbf{H} & \{d z(r) / d r\} \\
= & {\left[z(r)\left[J_{0}(k r)-j Y_{0}(k r)\right] r\right]_{0}^{\infty} } \\
& -\int_{0}^{\infty} z(r) \frac{d}{d r}\left\{\left[J_{0}(k r)-j Y_{0}(k r)\right] r\right\} d r \\
\simeq & -\int_{0}^{\infty} z(r) \frac{d}{d(k r)}\left\{\left[J_{0}(k r)-j J_{1}(k r)\right](k r)\right\} d r \\
= & -\int_{0}^{\infty} z(r)\left\{\left[-J_{1}(k r)-j J_{0}(k r)\right](k r)+J_{0}(k r)\right\} d r \\
\simeq & \int_{0}^{\infty} z(r)\left\{\left[j J_{0}(k r)+J_{1}(k r)\right](k r)\right\} d r \\
\simeq & 2 j k \mathbf{H}\{z(r)\} .
\end{aligned}
$$

In the latter proof, the bracket in the first line is equal to zero because of the additional conditions that we imposed, and the transition from the third to the fourth line is obtained with the help of

$$
\begin{aligned}
d J_{0}(x) / d x & =-J_{1}(x) \\
d\left(J_{1}(x) x\right) / d x & =J_{0}(x) x .
\end{aligned}
$$

The quality of those approximations is illustrated in Fig. 11. Fig. 11(a) represents the following function of the radius $r$ :

$$
z(r)=\sin (2 \pi r / 30) e^{-(1 / 2)(r-110 / 20)^{2}} .
$$

It presents a behavior similar to the observed ring-wave profiles. Fig. 11(b) shows its derivative. Following property (20), $j k \mathbf{H}\{z(r)\}$ is a good approximation of the complex Hankel transform of $d z(r) / d r$. From there, an estimate of $d z(r) / d r$ is found using property (18). It is represented in Fig. 11(c). Fig. 11(d) shows the-very small-difference between $d z(r) / d r$ and its estimate (with a vertical axis extended by a factor of 10). This example supports the use of the above approximations in the conditions of the experiments described in this paper.

\section{APPENDIX III}

\section{PROPAGATION OF CIRCULAR GRAVITY-CAPILLARY WAVES}

Following Lamb [21] and Le Méhauté [3], we assume that the motion is irrotational, allowing the definition of a potential function $\Phi(r, z, t)$, and a free surface elevation $\eta(r, t)$, where $r, z$, and $t$ are, respectively, the ring radius, the vertical (upward positive) coordinate, and the time from impact.

Let us recall the basic equations for circular surface waves on an incompressible irrotational fluid:

Mass conservation:

$$
\nabla^{2} \Phi=0
$$

Linearized kinematic boundary condition:

$$
\frac{\partial \eta}{\partial t}=-\frac{\partial \Phi}{\partial z} \mid z \simeq 0
$$

Linearized dynamic boundary condition:

$$
\frac{\partial \Phi}{\partial t}_{\mid z \simeq 0}=\eta g-\frac{\tau}{\rho}\left(\frac{\partial^{2} \eta}{\partial r^{2}}+\frac{1}{r} \frac{\partial \eta}{\partial r}\right)
$$

where $\tau$ is the surface tension, $\rho$ is the density of water, and $g$ is the acceleration of gravity.

If the water depth is very large compared to the wave amplitude and wavelength, a class of solutions of (21)-(23) is

$$
\begin{aligned}
\Phi(r, z, t)= & \left\{A \sin \left(\omega t+\alpha_{1}\right) J_{0}(k r)\right. \\
& \left.+B \sin \left(\omega t+\alpha_{2}\right) Y_{0}(k r)\right\} e^{k z} \\
\eta(r, t)= & \left\{A \cos \left(\omega t+\alpha_{1}\right) J_{0}(k r)\right. \\
& \left.+B \cos \left(\omega t+\alpha_{2}\right) Y_{0}(k r)\right\} k / \omega
\end{aligned}
$$

where the parameter $k$ is related to $\omega$ by the equation $\omega^{2}=$ $g k+\tau / \rho k^{3} . \alpha_{1}$ and $\alpha_{2}$ are arbitrary constants and $J_{0}$ and $Y_{0}$ are the first kind Bessel and Neumann functions of zeroth order.

It is important to note that the phenomena that appear just after impact are very complex [14] and certainly cannot be described by the linearized boundary conditions (22) and (23).

Those conditions are only valid at a distance from the source, where the wave height becomes small enough. They are fulfilled everywhere if we wait until the surface near the point of impact is again at rest (the observations show that this happens a few hundreds of milliseconds after impact).

From there, for the description of the waves far from the source $(r \gg 1 \mathrm{~cm})$, we kept the term containing the $Y_{0}$ function, even if this function presents a singularity at the origin $(r=0)$. 
From these solutions, we wish to hold only those corresponding to outgoing progressive waves; hence, for $r \rightarrow \infty$, the solution for a given wavenumber $k$ must be of the form

$$
\frac{F}{\sqrt{r}} \cos (\omega t-k r+\alpha)
$$

where $F$ and $\alpha$ are constants.

Considering the cosine and sine asymptotic approximations of $J_{0}$ and $Y_{0}$, it is possible to show that this condition is fulfilled if $\alpha_{2}=\alpha_{1}-\pi / 2$ and $B=A$.

If we represent the general solution by a continuous linear superposition of wave components characterized by a wavenumber $k$, it gives, with complex notations

$$
\begin{aligned}
\Phi(r, z, t)=\Re\left\{\int_{0}^{\infty}\right. & A_{1}(k) e^{-j \omega\left(t-t_{1}\right)} \\
& \left.\cdot\left[J_{0}(k r)+j Y_{0}(k r)\right] e^{k z} k d k\right\}
\end{aligned}
$$

and

$$
\begin{array}{r}
\eta(r, t)=\Re\left\{\int_{0}^{\infty} A_{0}(k) e^{-j \omega\left(t-t_{1}\right)}\right. \\
\left.\cdot\left[J_{0}(k r)+j Y_{0}(k r)\right] k d k\right\}
\end{array}
$$

where $\omega^{2}=g k+\tau / \rho k^{3}, A_{0}$ and $A_{1}$ are complex quantities, and $A_{0}(k)=-j A_{1}(k) k / \omega$. An estimate of $A_{0}(k)$ can be obtained from the height profile at time $t_{1}$ by solving (28) for $t=t_{1}$

$$
\eta\left(r, t_{1}\right)=\Re\left\{\int_{0}^{\infty} A_{0}(k)\left[J_{0}(k r)+j Y_{0}(k r)\right] k d k\right\} .
$$

We show in Appendix II that an approximate solution for $A_{0}(k)$ is given by

$$
A_{0}(k) \simeq 1 / 2 \int_{0}^{\infty} \eta\left(r, t_{1}\right)\left[J_{0}(k r)-j Y_{0}(k r)\right] r d r
$$

which corresponds to $\mathbf{H}\left\{\eta\left(r, t_{1}\right)\right\}$, the complex Hankel transform of $\eta\left(r, t_{1}\right)$, as we defined it in Appendix II.

\section{ACKNOWLEDGMENT}

The authors are grateful to Prof. F. Dupret (UCL) for many fruitful discussions about wave decomposition and vorticity.

\section{REFERENCES}

[1] M. Rein, "Phenomena of liquid drop impact on solid and liquid surfaces," Fluid Dynamics Res., vol. 24, no. 4, pp. 769-779, 1993.

[2] G. D. Crapper, Introduction to Water Waves. Chichester, U.K.: Wiley, 1984.

[3] B. Le Méhauté, "Gravity-capillarity rings generated by water drops," $J$. Fluid Mech., vol. 197, pp. 415-427, 1988.

[4] L. B. Wetzel, "On the theory of electromagnetic scattering from a raindrop splash," Radio Sci., vol. 25, pp. 1183-1197, 1990.

[5] P. Sobieski and F. L. Bliven, "Scatterometry of a drop impact on a salt water surface," Int. J. Remote Sensing, vol. 16, no. 14, pp. 2721-2726, 1995.

[6] P. Sobieski, L. F. Bliven, and C. Craeye, "Analysis of the radar backscattering of a salt water surface agitated by rain at scatterometric incidences," in Proc. 7th URSI Commission F Open Symp., Ahmedabad, India, 1995, pp. 168-170.

[7] R. K. Moore, Y. S. Yu, A. K. Fung, D. Kaneko, G. J. Dome, and R. E. Werp, "Preliminary study of rain effects on radar scattering from water surfaces," IEEE J. Oceanic Eng., vol. OE-4, pp. 31-32, 1979.
[8] F. L. Bliven, H. Branger, P. Sobieski, and J.-P. Giovanangeli, "An analysis of scatterometer returns from a water surface agitated by artificial rain: Evidence that ring-waves are the main feature," Int. J. Remote Sensing, vol. 14, no. 12, pp. 2315-2329, 1993.

[9] D. Atlas, "Footprints of storms on the sea: A view from spaceborne synthetic aperture radar," J. Geophys. Res., vol. 99, no. C4, pp. 7961-7969, 1994.

[10] C. Craeye, P. Sobieski, and F. L. Bliven, "Scattering by artificial wind and rain roughened water surfaces at oblique incidences," Int. J. Remote Sensing, vol. 18, no. 10, pp. 2241-2246, 1997.

[11] A. M. Worthington, A Study of Splashes. New York: MacMillan, 1963, reprint of a 1910 publication.

[12] B. Le Méhauté and S. Wang, "Spikes, domes and cavities," J. Int. Assoc. Hydraulic Res., vol. 5, pp. 583-602, 1987.

[13] J. Hallett and L. Christensen, "Splash and penetration of drops in water," J. de Recherches Atmospheriques, vol. 18, no. 4, pp. 225-242, 1984.

[14] E. G. Kilgore and J. A. Day, "Energy dissipation of water drops striking an undisturbed water surface," Tellus, vol. 15, no. 4, pp. 367-368, 1963.

[15] P. K. Wang and H. R. Pruppacher, "Acceleration to terminal velocity of cloud and raindrops," J. Appl. Meteorol., vol. 16, pp. 275-280, 1977.

[16] W. G. Van Dorn, "Boundary dissipation of oscillatory waves," J. Fluid Mech., vol. 24, pt. 4, pp. 769-280, 1966.

[17] O. Reynolds, "Papers on mechanical and physical subjects," J. Fluid Mech., vol. I, no. 86, 1900.

[18] M. Manton, "On the attenuation of sea waves by rain," Geophys. Fluid Dynamics, vol. 5, pp. 249-260, 1973.

[19] G. Chen, B. Chapron, J. Tournadre, K. Katsaros, and D. Vandemark, "Identification of possible wave damping by rain using TOPEX and TMR data," Remote Sensing of the Environment, vol. 63, pp. 40-48, 1998.

[20] R. Dautray and J.-L. Lions, Analyze Mathématique et Calcul Numérique pour les Sciences et les Techniques. Paris, France: Masson, 1987.

[21] H. Lamb, Hydrodynamics. New York: Cambridge Univ. Press, 1932.

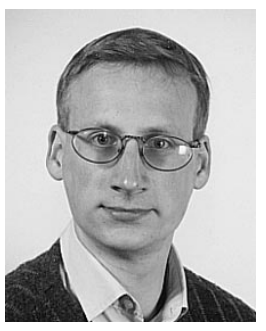

Christophe Craeye was born in Belgium in 1971 $\mathrm{He}$ received the degrees of electrical engineer and bachelor in philosophy in 1994 and the Ph.D. degree in 1998 from the Université Catholique de Louvain (UCL), Belgium.

Since 1994, he has been an Assistant at the Laboratoire de Télécommunications et Télédétection, UCL, Belgium. His research concerned the scattering of the sea surface in case of rain. He is now with the Technical University of Eindhoven, where he works on antenna arrays for radio-astronomic applications.

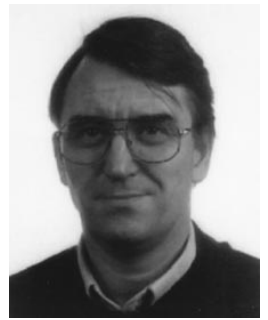

Piotr W. Sobieski (S'70-M'85) was born in Belgium in 1948. He received the degrees in electrical engineering in 1971, the Ph.D. degree in 1977, and Habilitation in 1987 from the Universite Catholique de Louvain (UCL), Belgium.

$\mathrm{He}$ is presently Professor at UCL (Laboratoire de Télécommunications et Télédétection), where he teaches fundamental electricity, telecommunications, and microwave remote sensing. Since 1995 , he has been the head of the Department of Electrical Engineering. Between 1971 and 1980, he was involved in propagation of microwaves through the atmosphere, particularly attenuation and depolarization of microwaves by hydrometeors. From 1980 to 1981, he stayed at the Technical Center (ESTEC) of the European Space Agency, The Netherlands, where his activities dealt with inverse scattering problems in microwave remote sensing. Between 1981 and 1990 , his main research activities concerned a unified approach of passive and active measurements, the development and validation of theoretical and numerical electromagnetic scattering models for the sea and the atmosphere, and synergic inversion of field data. From 1990 to 1991, he was a visiting Scientist at NASA Goddard Space Flight Center, where he worked on problems related to microwave remote sensing of rain above the ocean from space. Since 1991, he has worked at UCL on various remote sensing problems in cooperation with NASA (USA), ESA (NL), CETP (F), IMST (F), Ifremer (F), and other European laboratories. 


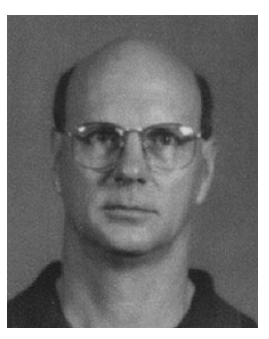

Larry F. Bliven received the B.S. degree in physics and the Ph.D. degree in marine sciences from North Carolina State University in 1971 and 1977, respectively.

His research primarily focuses on air-sea interaction processes and remote sensing of the oceans during storms and coastal storms. He is the Manager of the Rain-Sea Interaction Facility at NASA GSFC Wallops Flight Facility. See http://rsif.wff.nasa.gov

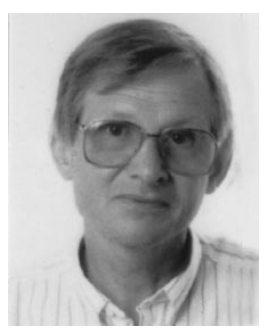

Albert Guissard (SM'71) received the degree of electrical engineer and the Ph.D. degree in applied sciences from the Université Catholique de Louvain (UCL), Belgium, in 1957 and 1965, respectively.

From 1961 to 1972, he was a Professor at Lovanium University, Zaire. In 1972, he joined, as Professor, the Telecommunication and Microwaves Laboratory (now Laboratoire de Télécommunications et Télédétection) of the Faculty of Applied Sciences, at UCL. He has taught fundamental electricity, electroacoustics, antennas and propagation, and microwave remote sensing. He has participated in research work in the field of nonlinear circuit theory, of basic problems in electromagnetism, of cylindrical antennas, and of attenuation and depolarization of microwaves by the atmosphere. Since 1978, he has been engaged in the field of microwave remote sensing. His research has concerned the observation of the oceans and of the atmosphere with radars, radiometers, and SAR's, along with the inversion of such measurements for the estimation of surface and atmospheric parameters. $\mathrm{He}$ is now engaged in research on vegetation and forest modelization and in the interpretation of polarimetric radar measurements.

Prof. Guissard is a member of URSI and the Remote Sensing Society. 